\title{
SESSION XI
}

\section{Challenges for Pediatric Radiology Using Computed Radiography}

\author{
Charles E. Willis, Bruce R. Parker, Michael Orand, and Milton L. Wagner
}

$\mathbf{T}$ HE CLINICAL EXPERIENCE base for pediatric Computed Radiography (CR) is even more limited than for general radiological practice. The generally small, but widely varying dimensions of pediatric patients, the lack of compliance, and our concerns about undue radiation exposure make these subjects especially challenging. Accommodation of inappropriate technique and postacquisition image processing, should make CR an ideal detector for pediatric examinations.

\section{MATERIALS AND METHODS}

Our hospital has been using CR in our outpatient pediatric care center since October 1995 . Our system initially consisted of an AGFA Diagnostic Console Model ADC70, PC-based Patient Identification (ID) Consoles, Sun-based Processing Station and Review Stations, a dedicated laser camera, and a DICOM PACS network including short and long-term archives and an RIS interface. We expanded our use of CR into our main radiology department in August 1997 with two additional CR devices, presently serving our emergency center.

\section{RESULTS AND DISCUSSION}

\section{Changes in Practice}

CR demands changes in practice by the technologist, radiologist, and referring physician. ${ }^{1} \mathrm{CR}$ involves changes in capture of the radiographic projection, development of the image, correction of suboptimal images, control of exposure factor, and interpretation of the image. Complicating factors include maintenance, quality control, technologist training, and fitful system maturation. As advocates of the technology, we embraced these challenges along with our partnership with the manufacturer.

\section{Performing the Examination}

With any new technology, documentation and standards lag behind fielding. Poor reliability is characteristic of new hardware models and soft-

From the Department of Radiology, Baylor College of Medicine, and Edward B. Singleton Department of Diagnostic Imaging, Texas Children's Hospital, Houston, TX.

Address reprint requests to Charles E. Willis, Ph.D., 6621

Fannin MC 2-2521, Houston, TX 77030-2399.

Copyright $\odot 1998$ by W.B. Saunders Company

0897-1889/98/1103-1047\$8.00/0 ware versions. Our CR device was the manufacturer's first in routine clinical operation in North America. Consequently, a maintenance support system was not adequately developed. Our devices underwent a succession of hardware and software upgrades, with interruptions in clinical services. We wisely maintained our capability to produce conventional screen-film images as a backup.

The ID console initially required manual entry of patient demographic data. When an RIS interface was added to our PACS network, the ID console could query the RIS interface to retrieve patient demographics, but still required manual entry of a six-digit Medical Record Number (MRN), which is our HIS unique patient identifier. Input errors render the image inaccessible to the radiologist, which can cause delay of diagnosis. Adding a Code 39 Bar Code Reader to each ID console, and scanning the bar code representation of the MRN on the paper examination request substantially reduced the number of misidentified images. A remaining problem is that without input, the ID console defaults to the previous MRN. This feature was deemed useful in expediting exams with multiple views. Unfortunately, this also means that images can be inadvertantly associated with a previous patient, and the error is very difficult to track down.

Post-acquisition processing by the technologist is necessary to verify correct orientation. This operation is performed at the processing station, which has many additional capabilities. Demographic information can be corrected. The image can be annotated if the lead letter marker is not visible within the collimator boundaries. If nondiagnostic, the image can be discarded before it is transmitted to the network servers. The appearance of sub-optimal images can be modified. Unfortunately, technologist changes of window/level are permanent and proved inappropriate for the radiologist. "Manual collimation," a powerful enhancement tool that disregards features outside a spatiallydefined region-of-interest, causes permanent modification that obscures the actual projected 
anatomy. This could hide pathology and prevents radiologist oversight of radiographic collimation.

Another powerful set of tools available on the processing station, but not on the review station, is Multispectral Image Contrast Adjustment (MUSICA). MUSICA is applied automatically to images based on examination. MUSICA parameters can be manually adjusted at the processing station. The operator has control over the magnitude of each MUSICA parameter, but not really the manner processing is distributed among small and large objects. The technologist has neither the time nor the training to exploit these functions. Tuning MUSICA values that are automatically applied is more productive.

Initially, simplified 8-bit images were transmitted from the processing station to the network. These images did not have sufficient grayscale resolution. For example, on a lateral view of the skull, nasal bones that were clearly visible on the processing station, could not be demonstrated on the review station using window and level tools. As soon as 12-bit images were transmitted to the network, all clinical features were visible including soft tissue.

Radiologists unanimously complained of "grainy" images. This is especially disturbing to radiologists, because they are trained to suspect images that have high levels of quantum mottle. Inadequate radiographic technique, improper calibration of the $\mathrm{CR}$, or inappropriate technique can cause grainy images. ${ }^{2}$ Multispectral decomposition of the raw image, processing the decomposed image, and recomposing the image can cause an inherent artifactual graininess, known as the "rice" artifact in wavelet image processing. ${ }^{3}$ One parameter, MUSI-contrast, boosts small objects and attenuates large objects. This also accentuates noise, a high frequency feature.

Autoranging is a feature that allows the CR to compensate for under- and over-exposure and produce an image with uniform density. Because CR does not produce an overly dark or light image, to monitor exposure factor control, you must interpret a numerical exposure index that is calculated during image acquisition. With our $\mathrm{CR}$, the value of "lgM" ( $\log$ of the Median value of the grayscale histogram) for an appropriately exposed image is $2.3 \pm 0.3$. Each increase of 0.3 in $1 \mathrm{gM}$ corresponds to a doubling of exposure. Our CR system also includes "dose monitoring" software. This software calculates an average $\operatorname{lgM}$ for the first 50 images of a specific exam type, view, and cassette size. Then it compares the $\operatorname{lgM}$ of the current image to the average by a thermometer graphic on the processing station and the laser film. Neither the lgM value nor the graphic is available at the review station.

A common misconception about $\mathrm{CR}$ is that exams should be performed without modifying the technique factors that were appropriate for a conventional screen-film detector. Although it is possible to produce an image with these techniques, the noise characteristics of CR approximate a 200 speed system and the $\mathrm{x}$-ray absorption characteristics are different from intensification screens in common use. Creating a new technique guide for every exam is a major project. Automatic technique guide generators have not proven useful for producing technical factors that are appropriate for CR.

The selection of cassette size is not without consequence in CR. ${ }^{4} \mathrm{CR}$ images are comprised of roughly the same number of pixels in the $\mathrm{x}$ and $\mathrm{y}$ dimensions, regardless of the size of the cassette. This causes several effects-the spatial resolution of smaller cassettes is better than larger cassettes, the quantum noise of smaller cassettes is worse than larger cassettes at the same exposure, and the magnification factor when initially displayed on the processing station or review station is greater for smaller cassettes than larger cassettes. While small cassettes are desired to visualize small structures, the cassette should be large enough to demonstrate the boundary of collimation. Some exams automatically assume collimation and failure to detect collimator edges can generate processing artifacts. Differential magnification complicates direct comparison of clinical features between two views, such as posterior-anterior and lateral, acquired with different-sized cassettes, and also between the same views current and prior studies where differentsized cassettes were chosen. This occurs quite frequently with pediatric patients whose sizes change rapidly.

\section{Interpreting the Images}

Radiologists have generally been pleased with image quality except for the graininess described above. The latter has been particularly bothersome in patients with interstitial lung disease and has led to overinterpretation of chest $\mathrm{CR}$ images (false positives).

CR bone images have been a problem in previous reports. ${ }^{5}$ While we have not performed a formal 
study, our radiologists have been very satisfied in the ability of the CR system to display subtle fractures and other pathologic lesions. The graininess does interfere, however, with proper evaluation of medullary bone, a particularly vexing problem in patients with metabolic and hematologic disorders.

CR images generally have more contrast than conventional images. We have found that it takes approximately three months for an experienced radiologist to reset his/her standards of normal to accommodate this change. Frustration levels are high during this period, but the radiologists must be coaxed into developing a new set of baselines. Once this is accomplished, radiologist satisfaction is quite high.

Some review station tools are not sufficiently developed for efficient use. For example, the measurement tool must be calibrated against an internal standard before realistic distances are reported. However, the pixel dimensions of every CR cassette are known and reported by the scanner: true measurements are made without calibration on images at the processing station, unfortunately, this information is either not shared or properly interpreted by the review station.

A definitive diagnosis often requires comparison to prior examinations. Our PACS system automatically retrieves (pre-fetch) relevant prior exams based on a set of rules. The rules can be based on modality and/or organ type. Considerable savings in radiologist time is achieved when the rules work properly, versus querying and retrieving prior exams from long term archive for each patient.

\section{Sharing the Images}

Acceptance of $\mathrm{CR}$ images by clinicians has been a pleasant surprise. They are particularly enthusiastic about the ability of a PACS to provide simultaneous viewing of images by clinician and radiologist for ease of consultation from geographically distant sites. Our system allows the radiologist to type in a "wet reading" (impressions) which is available to emergency center and outpatient physicians when they review their images. Uniform availability of images - no "lost films"-has made CR and PACS invaluable to the clinicians and radiologists. ${ }^{6}$ Acceptance by both radiologists and clinicians is dependent on thorough training in use of the system by a dedicated and motivated PACS support group.

As soon as exams are transmitted from the acquisition stations to the PACS network, they are automatically routed to multiple review stations. Autorouting can be based on patient location, modality, referring physician, organ type. In practice, autorouting by referring physician is not practical. Images from the same modality require different routing. Routing based on patient location is working for emergency center patients, but does not work well for outpatients. Routing by organ type would be ideal, except that the criteria is too specific, requiring an explicit match to the organ type which can have a number of valid permutations in our RIS. For successful autorouting by organ type, our system requires compilation of a huge study description table that addresses each permutation. This system could be manageable if the match criteria was less restrictive, or included a "wildcard" entry. Without a match, images are not automatically routed and must be retrieved. This problem also exists for prefetching.

\section{CONCLUSIONS}

Many of the challenges that we encountered may be overcome through experience, proper configuration of system, and further software enhancements. The overwhelming benefits of improved image access and immediate consultations create a demand for CR, even with sub-optimal images. The challenge for radiology departments is to execute a deliberate implementation of $\mathrm{CR}$, ensuring that we produce the best quality images without greater radiation dose to the patient.

\section{REFERENCES}

1. Willis CE, Mercier J, Patel M: Modification of conventional quality assurance procedures to accommodate computed radiography. Proceedings of the 13th Conference on Computer Applications in Radiology. Denver, CO, 1996, pp 275-280

2. Huda W, Slone RM, Belden CJ, et al: Mottle on computed radiographs of the chest in pediatric patients. Radiology 199:249252, 1996

3. Persons K, Palisson P, Manduca A, et al: An analytical look at the effects of compression on medical images. J Digit Imaging 10:60-66, 1997 (suppl)
4. Arthur RJ, Pease JN: Problems associated with digital luminescence radiography in the neonate and young infant. Pediatr Radiol 22:5-7, 1992

5. Cohen MD, Katz BP, Kalasinski LA, et al: Digital imaging with a photostimulable phosphor in the chest of newborns. Radiology 181:829-832, 1991

6. Don S, Albertina MJ, Ammann DL, et al: Soft-copy computed radiography in neonatal and pediatric intensive care units: Cost-savings analysis. Radiology 197:501-505, 1995 